Results Analysis produced three factor solutions for both the 'Causes' and 'Solutions'. These rich, shared accounts can be broadly summarised as: 'Causes' i) 'Unfair Society', ii) 'Individual Responsibility', iii) 'Hard Lives' and for 'Solutions' i) 'More than Money', ii) 'Guiding Choice', iii) 'Make Society Fair'.

No professionals were among respondents who exemplified (had a significant association with) 'Causes - Individual Responsibility' or 'Solutions - Guiding Choice' and no community participants exemplified 'Solutions - Make Society Fair'. There was an expected correlation between the 'Causes' and 'Solutions' factor solutions given the accounts identified.

Conclusion While there was some disagreement among professional participants, there was more of a focus on material, social and environmental factors. Community participants recognised a range of causes of worse health but even among those identifying structural causes as the main problem, structural solutions were not recognised. Despite the plurality of views there was broad agreement across the accounts about issues relating to money. While no easy solutions exist, addressing basic needs and the unpredictability of finances are seen as important for good health.

\section{RF24 ENGAGING UNDERREPRESENTED WOMEN AS PARTICIPANTS IN STUDIES ABOUT HEALTH RISKS: LIFE STAGE DIFFERENCES IN MOTIVATIONS TO PARTICIPATE}

${ }^{1}$ SJ Howcutt ${ }^{*}{ }^{2} \mathrm{~S}$ Barbosa-Boucas, ${ }^{3} \mathrm{AL}$ Barnett, 'LA Smith. 'Department of Midwifery, Community and Public Health, Oxford Brookes University, Oxford, UK; ${ }^{2}$ Department of Life Sciences, Brunel University, Uxbridge, UK; ${ }^{3}$ Department of Psychology, Health and Professional Development, Oxford Brookes University, Oxford, UK

\subsection{6/jech-2018-SSMabstracts.112}

Background Universal interventions to reduce health risks can widen health inequalities. Targeted approaches, where an intervention is tailored to specific groups, may overcome this limitation. Women aged 16 to 34 years with lower socioeconomic status are hard-to engage in research which limits our ability to understand how to address their health and lifestyle behaviours.

According to marketing research on spending, young adults aged 16 to 34 years pass through three main life stages: (1) dependency on older adults and individual interests, (2) leaving home and spending time with peers, (3) setting up home and having a family. The aim of this study was to evaluate how useful these life stage categories are for understanding women's motivations to participate in health research and for planning recruitment strategies.

Methods Nine focus groups with a total of 49 women (aged 16 to 34 years, without university education) were conducted. Women were recruited from different settings to mirror each of the three life stages above. Women in Further Education colleges represented category (1), women at work were for category (2) and mothers were for category (3). The focus groups explored participants' lifestyles, reasons for participating in the current study and beliefs about how research could be of value to them. Framework analysis was used to integrate theories from existing literature with themes emerging from the focus groups.

Results All women sought personal benefits from participation but these varied by life stage. Mothers wanted social opportunities away from caring responsibilities, while women at work and women in education valued monetary incentives more highly. A common theme across the groups was the desire to escape boring activities. Women's motivations to participate in health research were not purely altruistic but to improve their own social capital. There were differences in how women would choose to spend free time.

Conclusion The life stages were useful for understanding women's motivations to participate in health research. Advertising strategies to engage women aged 16 to 34 years in health research should be tailored to fit their life stages, their goals for free time and the value they place on participation. These findings suggest that different strategies are required to optimise recruitment across this age range. One recruitment strategy does not fit all.

\section{RF25 THE ROLE OF SOCIAL NETWORKS IN WEIGHT MANAGEMENT INTERVENTIONS DURING PREGNANCY AND POSTPARTUM FOR WOMEN WHO ARE OVERWEIGHT AND OBESE: A SYSTEMATIC REVIEW}

${ }^{1} B$ Bonello, 'L Matthews*, ${ }^{2} \mathrm{~J}$ Logue, ${ }^{1} S$ Simpson. ${ }^{1} M R C / C S O$ Social and Public Health Sciences Unit, University of Glasgow, Glasgow, UK; ${ }^{2}$ Institute of Cardiovascular and Medical Sciences, University of Glasgow, Glasgow, UK

\subsection{6/jech-2018-SSMabstracts.113}

Background Maternal obesity is a growing public health issue. It is associated with pregnancy and birth complications and increased risk for childhood and long-term obesity. Interventions focusing on individual behaviour change have had a modest impact on clinical outcomes. There is increasing evidence that social networks are an important driver of obesityrelated behaviours. The aim of this systematic review is to explore the effectiveness of lifestyle interventions that use social networks for weight management and to explore participants' experiences, and the process outcomes of these interventions.

Methods MEDLINE, PsychINFO, EMBASE, Cochrane, CENTRAL and CINAHL databases were searched as well as reference lists of included studies. Eligibility criteria included: intervention studies with a comparator group and qualitative studies/process evaluations of the included intervention studies; pregnant or postnatal women $(<2$ years) with $\mathrm{BMI}>25 \mathrm{~kg} / \mathrm{m}^{2}$; a lifestyle intervention with a social network component; and a gestational or postpartum weight outcome. Protocol papers were included to aid extraction of intervention components and behaviour change techniques (BCTs). Articles were screened by two independent reviewers. Data extraction is ongoing and studies will be assessed for quality and risk of bias. BCTs and social network functions are also being coded by two reviewers and will be summarised in tables. We will assess heterogeneity and, if possible, a random-effects meta-analysis and a priori specified subgroup analyses will be conducted. We will carry out a narrative synthesis.

Results A total of 10211 records were identified from databases with 73 full-text articles and another 25 identified from references screened. 30 articles (15 studies) were included. 8 potentially relevant studies are ongoing or results have not yet been published. Searches will be rerun before the final analyses. Preliminary findings are that the interventions are heterogeneous but most were group-based and delivered face-to-face. Interventions often advised on obtaining social support from existing social network (e.g. family), arranged support through created social networks 